\title{
A Study on Optimization Schemes in Subway Station by Anylogic
}

\author{
Yu Sun ${ }^{1, a}$ \\ ${ }^{1}$ School of Traffic and Transportation, Beijing Jiaotong University, Beijing, 100044, China \\ asunyu_bjtu@163.com
}

Keywords: Subway station; Optimization scheme; Passenger crowding; Anylogic software; Analytic Hierarchy Process

\begin{abstract}
As more and more passengers choose urban rail transit as their travel mode, many subway stations cannot satisfy the operation demand now because of the increasing passenger volume. In the peak hours, passenger crowding problem is serious in some subway station. This paper considers the practical conditions of a transfer subway station and makes three optimization schemes in the view of reconstruction engineering. The schemes are simulated by the Anylogic simulation software. Then an evaluation index system is built and the method of analytical hierarchy process is used to compare these schemes. The results show that compared with the measures of corner optimization and escalator addition, widening the existing staircase in the station is a better choice to relieve the transfer crowding. Furthermore, two suggestions are made to guide new stations design: one is that round corners should be used as much as possible; the other is that if the stay areas before the staircase or escalator zones are small, it is suggested to only set staircases rather than escalators.
\end{abstract}

\section{Introduction}

Urban rail transit has advantages of large volume, high speed, safety and punctuality, and more and more passengers begin to choose it as their travel mode[1]. So the subway station capacity is not enough for increasing passenger flow demand in many stations. Paper[2,3] planned some reconstruction schemes in the view of the real condition in subway station and the best scheme was selected. So we could found that effective optimization reconstruction schemes can relieve station crowding problems.

This paper made a survey of the transfer crowding condition in a transfer station of Beijing Subway in the morning peak hours and found out that the streamline transferring was in severe crowding condition, where average space for each person was close to the worse service level of $\mathrm{F}$ [4].According to the transfer streamline layout, the reason why crowding occurs will be found out. Then three optimization reconstruction schemes will be designed considering the station conditions in the view of engineering reconstruction. The schemes are simulated by the Anylogic software. Then an index system to evaluate these reconstruction schemes will be built. Finally, the assessment method of Analytic Hierarchy Process (AHP) is used to sort the schemes.

\section{The passengers crowding problem in a transfer station}

In this station, four transfer passages which are with the "L" shape are built. Passengers can pass through them in one direction only. In both transfer directions, the transfer ways are hall transfer and passage transfer. The plane illustration of paid areas in the station is shown in the Fig. 1, i.e. the red area is the transfer crowding area.

The causes of crowding here include the following:

(1)In the morning peak hours, the transfer passenger volume is larger than the design passing capacity of transfer staircase. The useful width of the transfer staircase is $2 \mathrm{~m}$. In $<$ Subway design standards> of GB50157-2013, the passing capacity for the staircase in the up direction with the width of $1 \mathrm{~m}$ is 3700 people/hour, so the design passing capacity for this transfer staircase is 7400 people/hour. By the survey mentioned before, the transfer passenger volume from Line 4 to Line 2 is 8152 people/hour, which is larger than its design passing capacity. 
(2)The transfer staircase is set as in the up direction. When passengers climb so long a staircase, they will consume lots of physical power and slow down their walking speed. So the real passing capacity is smaller than the designed one.

(3)There exist conflicts in the streamline joint of the two transfer passages. The two transfer passages and the two staircases contain corners of 90 degrees, where if streamlines join together, conflicts will occur due to avoiding crash, slowing down or surpassing the former passengers because of change of walking speed and passage width[5].

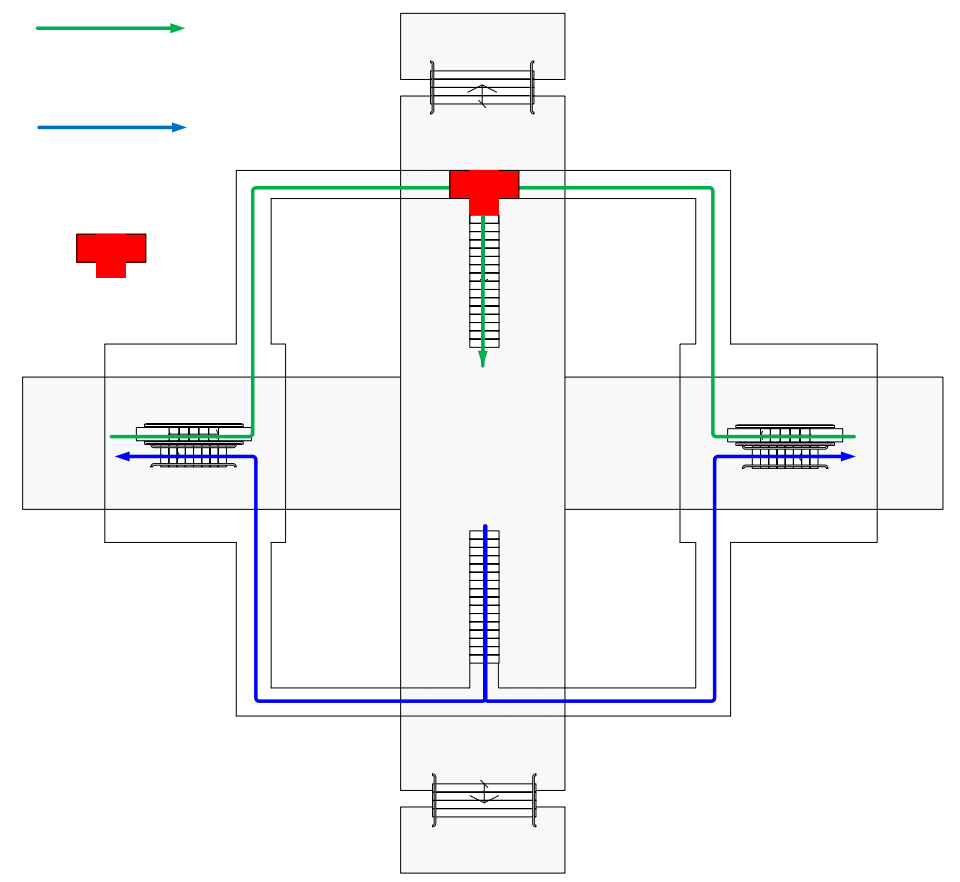

Fig. 1 The plane illustration of paid area in the transfer station

\section{Optimization schemes of transfer streamline in the transfer Station}

The optimization schemes of transfer streamline will be designed in the view of station engineering reconstruction.

Scheme 1: Corner optimization.

Turn the right-angle corners of the joint connecting transfer passage and transfer staircase to round corners with the radius of $2 \mathrm{~m}$, which can smooth the passenger turning process by decreasing friction conflicts, as is shown in Fig 2.

Scheme 2: Widening the staircase width.

Widen the transfer staircase by $1.4 \mathrm{~m}$, i.e. $0.7 \mathrm{~m}$ at each side, as is shown in the Fig. 3.

Scheme 3: Adding an escalator.

Add an escalator with the width of $1 \mathrm{~m}$ and the speed of $0.5 \mathrm{~m} / \mathrm{s}$ in the up direction (Fig. 4).

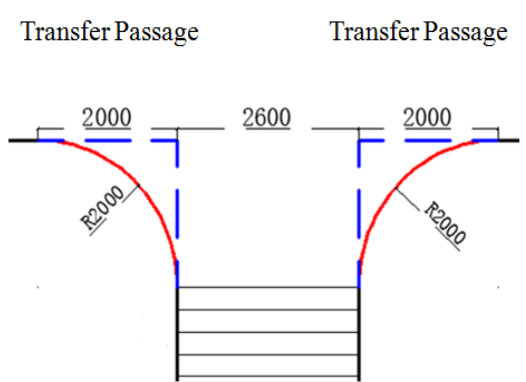

Fig 2 Corner optimization
Transfer Passage Transfer Passage

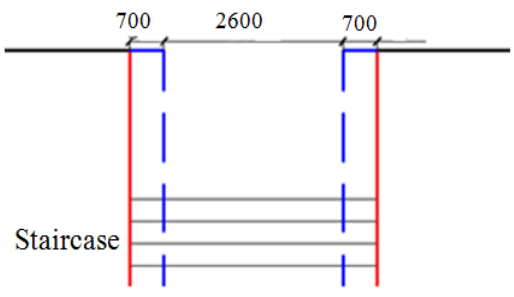

Fig. 3 Widen the staircase 


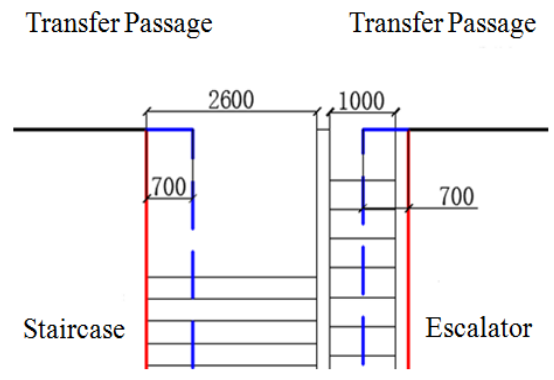

Fig. 4 Add an escalator

\section{Comparison of optimization schemes in the transfer station}

The scheme evaluation index system. The principle of station reconstruction is to cost less to get a better optimization result, so the coefficients are classified into two kinds which are cost coefficients and effect coefficients. The cost indexes include fare cost and time cost. This paper considers the real condition and chooses reconstruction cycle as the cost indexes, and maximum passenger density in area and average passenger transfer time as effect indexes.

The cost coefficients in the schemes can be calculated due to the construction organization design and the construction drawing in relevant Beijing Subway standards. This paper is in the first stage of scheme making, it is allowed to simplify the calculation process of scheme cost indexes and only refer to engineering experiences.

As to scheme effect coefficients, maximum passenger density in area focuses on the area where density is the maximum in the streamline. Average passenger transfer time is the average value of passengers walking from the staircase or escalator of Line 4 to the platform of Line 2 . The values of these two effect coefficients can be calculated in the station after reconstruction in the simulation module of Anylogic software [6].

Index calculation of each scheme. Cost indexes are calculated using engineering experiences as a reference here and the station after reconstruction is simulated and modeled in Anylogic software to get the effect indexes.

(1)Estimation of cost indexes in each scheme

Only the reconstruction cycle was selected as the cost indexes because the subway station is in operating, it is more important to complete the scheme quickly than save the fare cost. So the fare cost index is excluded.

(2)Calculation of effect indexes in each scheme

The passenger module in Anylogic software is used to model and simulate the zone from the staircase and escalator in the hall of Line 4 to the platform of Line 2, as is shown in the Fig. 5, where the color for the joint of the transfer passage and staircase is close to deep red meaning the crowding condition in this area is quite severe and there exists great potential safety threat here.

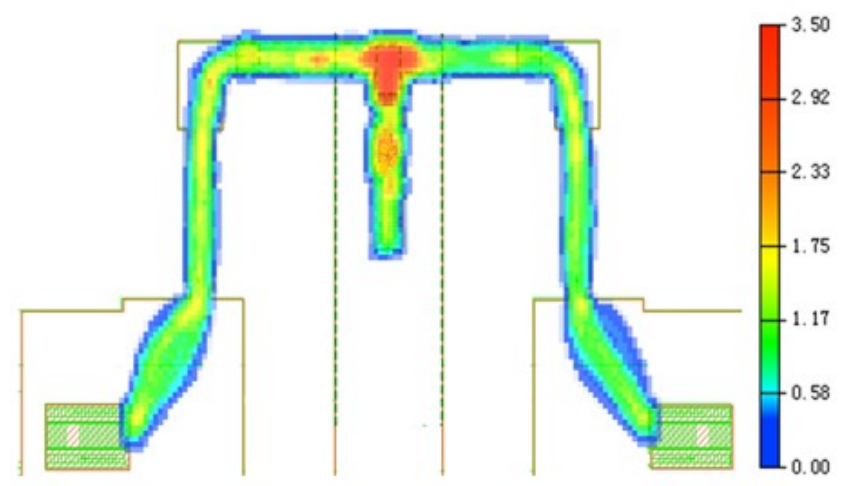

Fig. 5 Simulation illustration of the condition where passengers are crowed

When each scheme is simulated, the input data are set as the same as the original simulation. The effect indexes data could be calculated from the software. 
Detailed index descriptions in each scheme are as shown in the following Table 1.

Table 1 Index descriptions in each scheme

\begin{tabular}{cccc}
\hline Scheme & $\begin{array}{c}\text { Reconstruction } \\
\text { cycle [months] }\end{array}$ & $\begin{array}{c}\text { Maximum density } \\
\text { in area [people/m }\end{array}$ & $\begin{array}{c}\text { Average transfer } \\
\text { time [s] }\end{array}$ \\
\hline One & 1 & 2.73 & 113.8 \\
\hline Two & 2.5 & 1.97 & 104.2 \\
\hline Three & 3 & 2.18 & 103.5 \\
\hline
\end{tabular}

Comparison results of the optimization schemes. AHP can analyze the influencing factors in each layer, evaluate them in quantity and change them in a standard way so as to sort the optimization schemes quickly and effectively[7].

Expert evaluation method is used to decide the judge matrix and then square root method is adopted to calculate the index weights. Finally consistency test is made. The weights of the objective are in the Table 2.

\begin{tabular}{cccc} 
Table 2 & \multicolumn{4}{c}{ Weights of each scheme } \\
\hline Scheme & One & Two & Three \\
\hline Weight & 0.268 & 0.394 & 0.338 \\
\hline
\end{tabular}

According to the upper table, the rank of schemes is: Scheme $2>$ Scheme $3>$ Scheme 1 . The result shows that increase the width is better than optimize the corner, but another thing need to be noticed is width the staircase is better than add an escalator. The reason is many passengers choose the escalator to save strength lead to the crowding problem before the escalator.

\section{Summary}

This paper researches on the crowding problem in a transfer station in the morning peak hours. Three transfer optimization reconstruction schemes are designed. The assessment method of AHP is used to sort the schemes. The optimal scheme to relieve the crowding problem is to widen the width of the transfer staircase in this station.

Finally, there are two suggestions made in this research to guide transfer passage design in new station constructions. The first one is that more round corners should be designed to smooth the passenger turning process when new stations are constructed. The second is that when staircases or escalators need to be installed in the passages, sometimes only staircases should be set because many passengers intend to use escalators to save physical strength but the area is too small to hold the people, so the area before escalator could appear crowded phenomenon which is likely to cause danger.

\section{References:}

[1]Baohua Mao, Mingjun Liu, Rong Huang and Peng Du. (2011). Operation theories and key technologies of rail transit networks. Beijing: Science Press. (In Chinese)

[2]Jia He and Shumin Hou (2004). Tianjin metro station reconstruction design. Urban Rapid Rail Transit. 17:92-95. (In Chinese)

[3]Haoling Wu and Huixuan Li (2012). On the optimum schemes of transfer lines at Xizhimen Station of Beijing Metro. Urban Mass Transit. 15(1):88-92. (In Chinese)

[4]National Transportation Safety Board (2009). Transit capacity and quality of service. China Architecture \& Building Press

[5]Li Zhao (2011). Research on traffic design theory and methods of urban rail transit hubs. Beijing: Beijing Jiaotong University. (In Chinese)

[6]Liyang Chen, Rui Song, Zhijie Li and Tingting Li. (2013). Simulation study on the layout of metro station hall facilities based on Anylogic. (In Chinese) 
[7]Li Zhu, Haiguang Tian and Yong Yang (2014). Analysis on application of AHP method in selection of cut-and-cover method. Tunnel Construction. 34(3):243-248. (In Chinese) 